\title{
DiE STROMNAI DES POLYKRATES VON SAMOS
}

\author{
F. X. Ryan
}

\begin{abstract}
Athenaios retails a story, little heeded, but variously understood when not misunderstood, according to which Polykrates of Samos was a purveyor of stromnai while still a private citizen. This paper offers two new explanations of the word stromnai, gives some reasons for attaching credence to the report, and surmises that the original version of the story preserved by Athenaios made Polykrates responsible locally for innovations also seen elsewhere.
\end{abstract}

Es heißt, Polykrates von Samos habe vor seiner Alleinherrschaft Gegenstände herstellen lassen und sie dann denjenigen, die eine Hochzeit oder größere Empfänge veran-

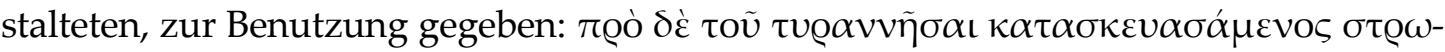

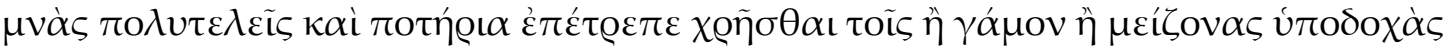

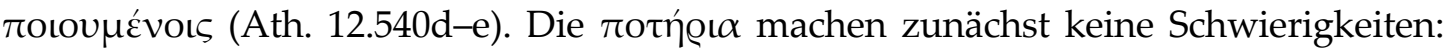
Das sind Trinkgefäße, in denen mit Wasser vermischter Wein serviert wurde. Dagegen bereiten die kostspieligen $\sigma \tau \varrho \omega \mu \nu \alpha i ́$ einiges Kopfzerbrechen. Die althistorische Forschung hat diesem Bericht wenig Glauben geschenkt ${ }^{1}$ und sich mit ihm deshalb nicht gründlich befaßt. ${ }^{2}$ Von den Althistorikern und Philologen, die die sehr teuren $\sigma \tau \varrho \omega \mu v \alpha i ́$ trotzdem erwähnten, scheint keiner bisher bemerkt zu haben, daß das Wort verschiedentlich übersetzt bzw. gedeutet wird. So begegnen uns in der Sekundärliteratur bzw. bei der Wiedergabe der Stelle „hangings" bzw. „Wandbehänge," ", ${ }^{\prime \prime}$ "arpet" bzw. „Tep-

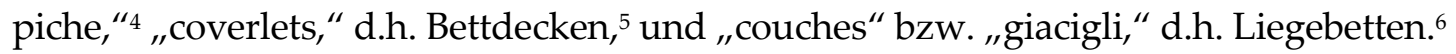

\footnotetext{
${ }^{1}$ Statt aller s. Lenschau (1952: 1727): „Wenn der bei Athenaios genannte samische Chronist die Sache so darstellte, als ob P(olykrates) vor seiner Tyrannis eine Art Tafelverleihinstitut betrieben habe, so braucht man das ebensowenig ernst zu nehmen wie die Vermutung Ures, die daraufhin annimmt, daß P(olykrates) schon eine leitende Rolle in der samischen Metall- und Wollindustrie gespielt habe, ehe er sich den Weg zur Macht bahnte."

${ }^{2}$ Plaß (1859: 243) berief sich auf Athenaios und stellte fest, ohne näher darauf einzugehen, daß „ein sehr freigiebiger Gebrauch seiner Schätze ihm...Popularität gewinnen mußte“; Berve (1967: 107) scheint das Thema zu streifen, indem er schreibt, daß Polykrates „eine Hetairie von Standesgenossen...angeblich durch große Freigiebigkeit für sich gewann“; Shipley (1987: 82) gönnt der Geschichte nur einen flüchtigen Blick: „Athenaeus goes on to tell the story of Polycrates' carpet-hire service..."; bei de Libero (1996) scheint die Episode ganz zu fehlen. - Auch bei Thompson (1988: 15) wird behauptet, Polykrates „had begun making his money by renting out" die Gegenstände. Diese Vermutung findet aber im griechischen Text keine Stütze. Neben Plaß sprechen schon Rawlinson et al. (1862: 365 A. 9) richtigerweise von "the measures whereby he attained...popularity“; s. auch Sewell (1862: 123): „in order still further to increase his popularity.”

${ }^{3}$ Drei englischsprachige Autoren verwenden den Ausdruck „his rich hangings": Rawlinson et al. 1862: 365 A. 9, Sewell 1862: 123, Harrison 1893: 221-22. Friedrich (2000: 141) übersetzt „wertvolle Wandbehänge.“ Vielleicht gehören hierher auch die „sumptuous draperies“ von Gulick (1933: 445).

${ }^{4}$ Shipley 1987: 82, Lenschau 1952: 1727.

${ }^{5}$ Ure 1922: 79, Thompson 1988: 15.

${ }^{6}$ Yonge (1854: 865): „,costly couches“; Fornara (1983: 34): „,expensive couches“; Galvagno (1994: 27): „,sontuosi giacigli."
} 


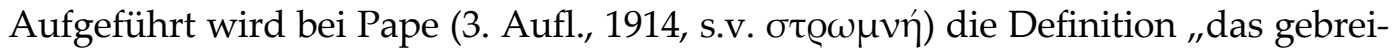
tete oder bereitete Lager" (d.h. Bett), während Liddell-Scott-Jones (9th ed., 1940, s.v.) die Definitionen „,bed spread or prepared: generally, bed, couch“ und „mattress, bed-

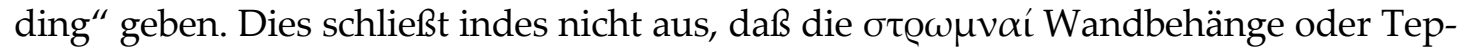

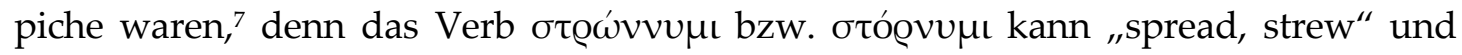
„spread smooth“ (LSJ, s.v.) bedeuten und man muß daher mit der Möglichkeit rechnen, daß das Substantiv alles bezeichnet, was gebreitet und vielleicht auch drapiert

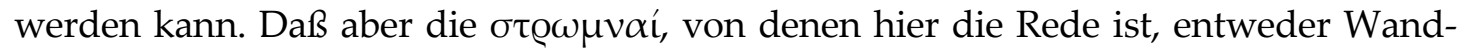
behänge oder Teppiche waren, läßt sich aus dem Grund verneinen, daß sie bei einem Symposium von sekundärer Bedeutung waren. ${ }^{8}$ So wird beispielsweise die Ausstat-

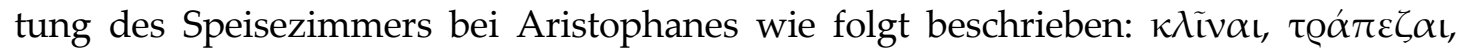

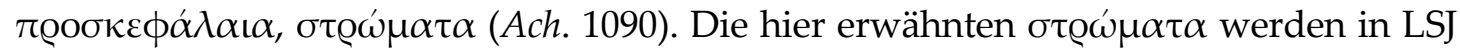
(s.v.) als "pl., bedclothes, coverings of a dinner-couch“ definiert, was korrekt zu sein scheint. ${ }^{9}$ Auf Wandbehänge und Teppiche konnte man also verzichten. ${ }^{10}$ Da der überlieferte Text des Athenaios nur zwei Arten von Gegenständen erwähnt bzw. der Er-

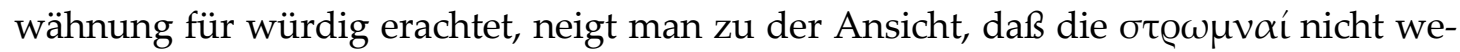

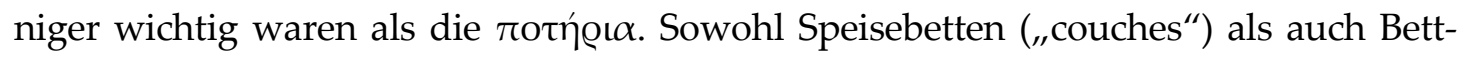
decken („,coverlets“) werden in das knappe Verzeichnis des Aristophanes aufgenommen und erfüllen somit diese Bedingung. Die Vasenbilder lehren, daß letztere keine Bettenbehänge ${ }^{11}$ oder Betttücher ${ }^{12}$ waren, sondern wirkliche Decken, in die sich der Speisende bzw. Trinkende hüllte. ${ }^{13}$

Über die sozialen und wirtschaftlichen Verhältnisse in Samos um 540 v. Chr. sind wir natürlich nur sehr dürftig unterrichtet. Wem genau Polykrates diese Gegenstände ausgeliehen haben soll, ob das seine Standesgenossen oder einfachere Menschen

\footnotetext{
${ }^{7}$ Langenscheidts Taschenwörterbuch der griechischen und deutschen Sprache (1986, s.v.) listet neben „Lager" und „Bett“ die Definitionen „Teppich,“ „Decke“ und „Polster“ auf.

${ }^{8} \mathrm{Ab}$ dem späten 5. Jhdt. werden Teppiche jedoch wenig Verwendung gefunden haben, denn durch sie wären die Bodenmosaiken aus der Sicht verschwunden. Zum Aufkommen griechischer Kieselmosaiken s. Westgate (1998: 93-95, 97, 102), die betont, daß sie in klassischer Zeit den andron (Männersaal) kennzeichnen. Was Wandbehänge anbelangt, so werden sie in dem Maße an Bedeutung verloren haben, wie die Wanddekoration an Bedeutung gewann. Zum Wandputz und zu Wandmalereien in Wohnhäusern, die anfangs ebenfalls mit dem andron verquickt waren und in hellenistischer Zeit häufiger wurden, s. Westgate 1998: 100. - Es kommt nicht von ungefähr, daß Mosaiken „waterproof and easy to clean“ waren (ebd. 97). Bedenkt man dies, so darf man schließen, daß Teppiche nicht einmal in archaischer Zeit eine große Rolle spielten.

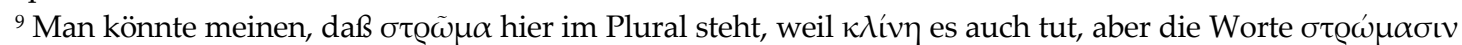

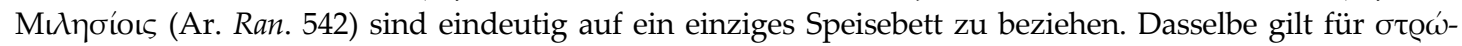
$\mu \alpha \tau \alpha \pi \mathrm{o} \lambda v \tau \varepsilon \lambda \tilde{\eta}$ (Athen. 2.48e) und $\sigma \tau \varrho \omega \mu \nu \alpha i ̃ \varsigma ~ \pi \mathrm{o} \lambda v \alpha v \theta \varepsilon \dot{\sigma} \sigma$ (Diod. 31.8.12).

${ }^{10}$ Entsprechendes findet sich in anderen Schriften. Vössing (2004: 116) faßt zusammen, was Diod. 21.12.4-6 über den hellenistischen Bankettluxus lehrt: „in erster Linie ging es um Betten, Polster, Tische und Geschirr."

${ }^{11}$ Aber "on the monuments one sometimes sees a valance hanging from the edge of the bed-frame, half way or all the way down," wie Richter (1966: 54) bemerkt.

12 Vgl. Richter (1966: 53): „Greek writers of the sixth and fifth centuries do not refer to sheets.“

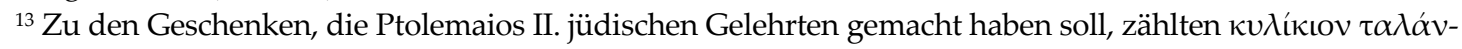

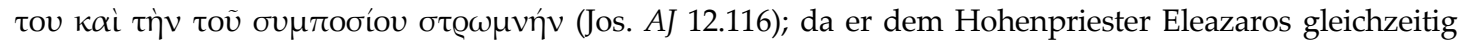

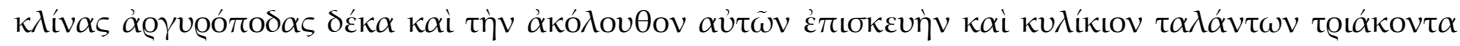

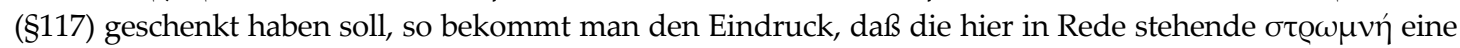
Decke war.
} 


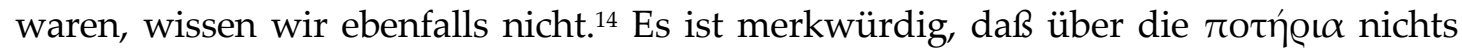
näheres berichtet wird: Da fällt einem ein, daß diese Becher möglicherweise nicht aus Edelmetall gefertigt waren und deren Benutzer vielleicht keine eigenen hatten. Wenn aber die $\sigma \tau \varrho \omega \mu v \alpha i ́$ mit $\pi 0 \lambda v \tau \varepsilon \lambda \varepsilon i \check{\iota}$ explizit als aufwendig bezeichnet werden, erscheint es möglich, daß die Benutzer doch eigene hatten, aber keine so guten. ${ }^{15}$ Aristophanes scheint uns jetzt im Stich zu lassen, denn er hätte Speisesofas $\kappa \lambda \tilde{i} \alpha \alpha$ u und Sofadecken $\sigma \tau \varrho \omega \mu \alpha \tau \alpha$ genannt. Vielleicht steht man aber nicht vor der Wahl zwischen Speise-

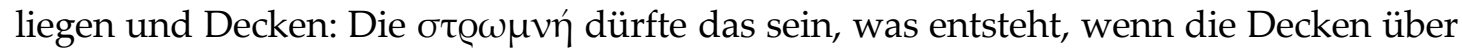
das Speisebett gebreitet werden. Es spricht nicht dagegen, daß neben dem Ausdruck

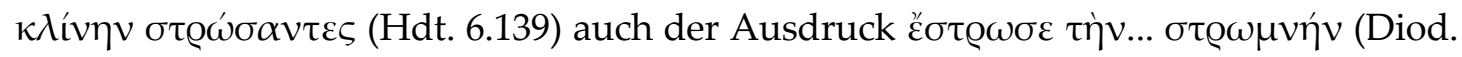
21.12.4) vorkommt, denn bei letzterem wird das Ergebnis des Vorgangs einfach vorweggenommen. ${ }^{16}$ Es ist also sehr wohl möglich, daß die uns interessierenden $\sigma \tau \varrho \omega-$ $\mu v \alpha i ́$ als „bedeckte Speisebetten“ aufzufassen sind. Die Benutzer werden sicherlich auch Tische nötig gehabt haben. Letztere durften nicht fehlen: Der älteste Beleg für die Sitte des Lagerns beim Mahle erwähnt sie in gleichem Atemzug mit den Klinen: $\kappa \lambda \tilde{i} v \alpha \iota$

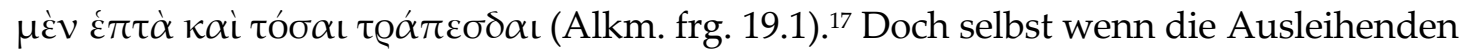
normalerweise beim Mahle saßen, müßten sie eigene Tische besessen haben. ${ }^{18}$ So überrascht es nicht, daß Polykrates keine Tische zur Verfügung gestellt haben soll.

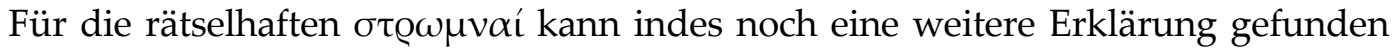
werden. Das Wort $\sigma \tau \varrho \omega \mu \nu \eta$, welches vielfach ein Bett bezeichnet (Aischyl. Ch. 671, Eur. Phoin. 421, Thuc. 8.81.3, Xen. Symp. 4.38), benennt an einigen Stellen nur einen Teil

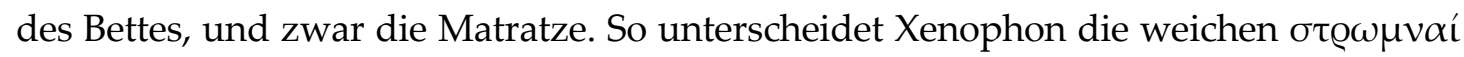

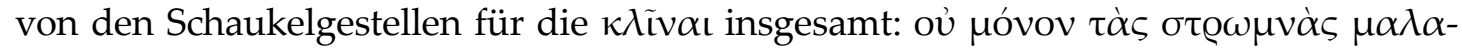

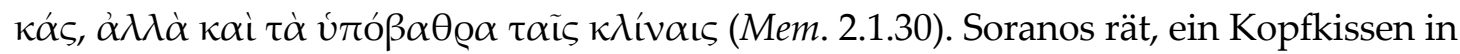
eine konkave Matratze zu verwandeln und das neugeborene Kind auf diese zur Ruhe

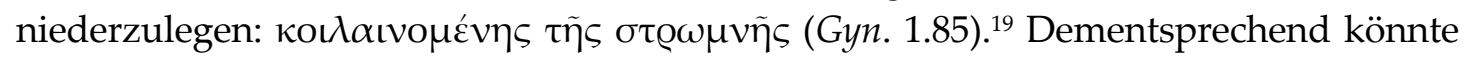
es sich bei den hier in Rede stehenden $\sigma \tau \varrho \omega \mu \nu \alpha i ́$ um Polster handeln. Es versteht sich von selbst, daß weiche Polster bei einem stundenlangen Symposium nicht unwesentlich waren. Schon einer der frühesten Belege für den Brauch, im Liegen zu speisen, spricht

\footnotetext{
${ }^{14}$ Zweifelsohne war das Symposion in der Regel „Medium der sozialen Bindung zwischen den einzelnen Aristokraten“ (Stahl 1987: 26), es könnte aber hier eine bestätigende Ausnahme vorliegen. Man darf aber nicht als gesichert ansehen, daß Polykrates so oder so bestrebt war, bei mittleren Schichten Eindruck zu schinden. Man muß sich nämlich davor hüten anzunehmen, daß seine Standesgenossen für Symposia gut gerüstet waren.

${ }^{15}$ Dentzer (1982: 446) behauptet von „les coupes“ bei Banketten: „Elles ne manquent jamais dans les distri-

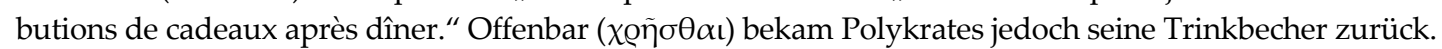

${ }^{16}$ Es sei denn, daß hier $\sigma \tau \varrho \omega \mu v \eta ́$ für $\sigma \tau \varrho \omega ́ \mu \alpha \tau \alpha$ steht; s. A. 26 unten.

${ }^{17}$ Vgl. dazu Boardman (1990: 124): „Whether this is a Spartan wedding feast or a recollection of Alcman's early life in Lydian Sardis, or a combination of both, we cannot say.“ Boardman (ebd. 124) schreibt: „There is a possible indication of reclining for a feast earlier in the century, in the Ephesian poet Callinus“; über das lange Bruchstück von Kallinos urteilte noch Gerber (1997: 100): „It seems probable in view of the opening $\kappa \alpha \tau \alpha ́ \kappa \varepsilon เ \sigma \theta \varepsilon$ that Callinus sang these verses at a symposium, the occasion on which so much elegiac

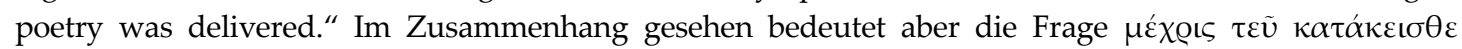
(Kallin. fr. 1.1) nicht, daß seine Landsleute bei Tisch lagen, sondern daß sie untätig herumlagen. So übersertzte Müller (1882: 180): „Wie lange liegt ihr in Ruhe da...?“, und inzwischen Miller (1996: 20): „How long are you lying idle?"

${ }^{18}$ Vgl. Richter (1966: 64): „When women are eating they sit in front of a table.... And this would apply to the simpler Greek people."

${ }^{19}$ Dazu s. Ploß 1884: 59.
} 


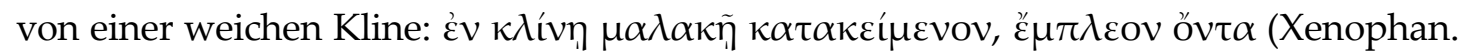
frg. 22.1).

Nur eine der beiden Alternativen kann zutreffen. Ein Gedicht Theokrits dürfte einen Fingerzeig geben. ${ }^{20}$ Sein aus den 270er Jahren stammendes fünfzehntes Idyll thematisiert das Adonisfest im königlichen Palast des ptolemäischen Alexandrien. Im Lied der Sängerin (Zz. 100-144) wird die Kline des Gottes beschrieben. Das Sofagestell war offenbar aus Ebenholz gemacht und mit Gold und kleinen Elfenbeinskulpturen verziert (Zz. 123-124). ${ }^{21}$ Bei A. S. F. Gow fährt die Argiverin folgendermaßen fort:

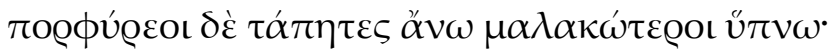

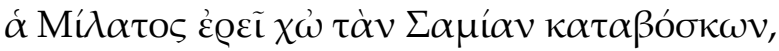

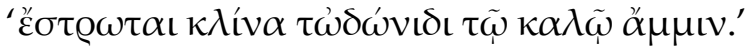

(Theokr. 15.125-127)

Gow übersetzt:

And crimson coverlets above, as soft as sleep.

Miletus will say, and he who pastures Samos with his flocks,

'Ours are the coverlets for the fair Adonis couch.'22

Neuere englische Übersetzungen weichen davon wenig ab. ${ }^{23}$ Burton hat richtig erkannt, daß der Dichter ganz bewußt diese beiden Orte hervorhob: „A reference to Samos and Miletus, recent acquisitions of the Ptolemaic kingdom, would compliment the royal house. ${ }^{\prime 24}$ Der samische Hirte ist aber so nicht gut integriert, denn hier macht Miletus nur mit der Hilfe eines Samiers, was die Stadt sonst ganz allein schaft: Miletus war ja für ihre Schafzucht (Ath. 12.540d) und ihren Wollstoff bekannt. Es ist kaum ausschlaggebend, daß die Scholien auf die schönen $\sigma \tau \varrho \omega \dot{\mu} \mu \tau \tau \alpha$ beider Orte und $\tau \dot{\alpha}$

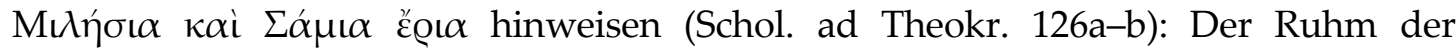
samischen Wolldecken dürfte aus dem zu besprechenden Text selbst gefolgert worden sein. Eine Aufgabe bekommt der Hirte aber in der Übersetzung von E. Staiger:

Purpurfarbene Decken darüber. ,Weicher als Schlummer,'

Wird die Milesierin sagen, und der da weidet auf Samos:

,Die für den schönen Adonis gebreiteten Kissen sind unsre!' ${ }^{25}$

Staigers Auffassung des Textes ist offenkundig richtig. Miletos ist jetzt für die Decken ( $\tau \alpha \dot{\pi} \pi \eta \tau \varepsilon \varsigma \alpha ̆ v \omega)$ alleine zuständig, was auch anderweitig bezeugt ist: So werden

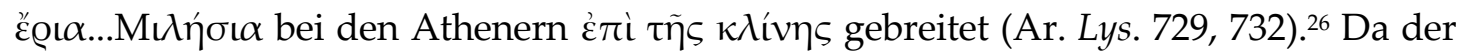
Dichter der Abwechslung halber nicht $\alpha$ $\Sigma \alpha ́ \mu \circlearrowleft \varsigma$ schreibt, sondern von einem Hirten

\footnotetext{
${ }^{20}$ Schon Ure (1922: 79) zog Theokrit zu Rate bei seiner Erklärung des Wortes: „A form of the corresponding verb ( $\left(\sigma_{\tau} \varrho \omega \tau \alpha \mathrm{l}\right)$ is used by Theocritus in the passage where he refers to the famous wools of Samos and Miletus."

${ }^{21} \mathrm{Vgl}$. dazu Boardman (1990: 126): „pieces of inlaid ivory from kline legs have been found in Athenian and South Russian graves, while there are also many very explicit depictions of them in painting or relief."

22 Gow 1952a: 118-19.

${ }^{23}$ Burton (1995: 172) übersetzt: „And the purple coverlets above, softer than sleep; / Miletus will say and the shepherd who herds sheep in Samos, / , the couch covered for fair Adonis is our work. “"; Verity (2002: 48) übersetzt: „And the couch's red coverlets, softer than sleep! / Milesian women and shepherds of Samos will say, / ,We made the coverlets for beautiful Adonis' couch."

${ }^{24}$ Burton 1995: 143.

25 Staiger 1970: 109.

${ }^{26}$ Hierauf bezieht sich wohl der byzantinische Dichter, selbst wenn er das Wort $\sigma \tau \varrho \omega \mu v \alpha i ́$ gebraucht: $\pi \varepsilon \varrho i$

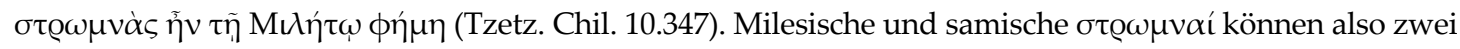
verschiedene Dinge sein.
} 
spricht, ist es ausgeschlossen, daß Samos für das Ebenholz, das Gold oder das Elfenbein sorgte. In diesem Zusammenhang kann $\kappa \lambda i ́ v \alpha$ also nur die Sofapolster bedeuten. Gow ersetzte das in Zeile 127 überlieferte $\ddot{\alpha} \lambda \lambda \alpha$ durch das Pronomen $\ddot{\alpha} \mu \mu \mathrm{t} v$, weil er hier Miletos und den samischen Hirten zusammen sprechen läßt, ${ }^{27}$ aber wenn man diese Aussage einzig dem Hirten in den Mund legt, dann spricht er stellvertretend für alle samischen Hirten, wie Staiger richtig erkannte. Der Auftritt des Hirten legt nahe, daß sein Berufsstand nicht nur die Polsterüberzüge, sondern auch die Polsterfüllung erzeugt hatte. Für die Matratze des neugeborenen Kindes war ein mit Wollflocken aus-

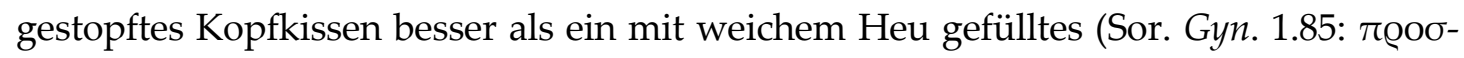

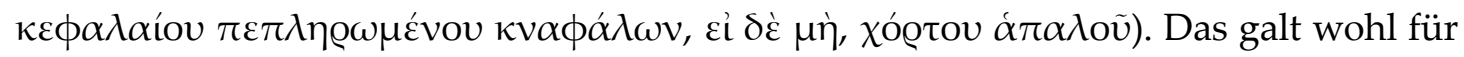
mythologische Gestalten auch.

Wenn nicht alles trügt, war Samos zur Regierungszeit des Ptolemaios II. für ihre

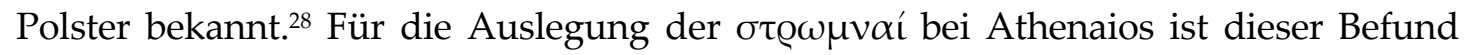
nicht ohne Belang, denn die fraglichen Luxusartikel sollen nicht aus dem Ausland im-

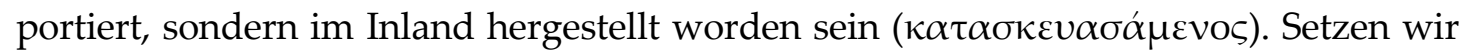
einmal voraus, daß die Geschichte von den ausgeliehenen $\sigma \tau \varrho \omega \mu v \alpha$ í glatt erfunden ist,

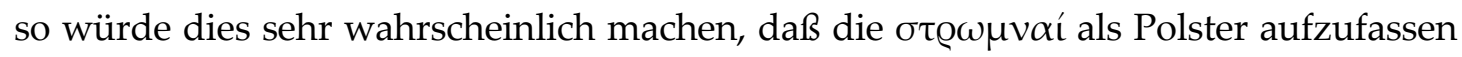
sind. Da der Samier Polykrates für seinen luxuriösen Lebensstil berühmt und berüchtigt war, hätten die meisten antiken Schriftsteller es für selbstverständlich gehalten, daß er über die prunkvollen samischen Polster verfügte. Sie hätten sich nicht gefragt, ob die angenommene chronologische Reihenfolge zutreffend ist oder ob die Polster erst in der Zeit nach Polykrates zum Vorschein kamen. Einige dieser Schriftsteller hätten es sogar fertig gebracht, die kostbaren Polster unbekümmert auf den dekadenten Polykrates zurückzuführen. Es ist dann durchaus möglich, daß Polykrates erst im Rahmen einer Aitiologie der Polster etwas mit ihnen zu tun bekam. Nehmen wir hingegen an, daß die

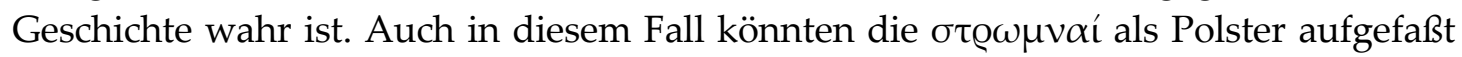
werden, denn solche Polster sind offenbar kein Hirngespinst. Darunter könnte man aber auch bedeckte Speisebetten verstehen.

Der Bericht von Athenaios vermittelt den Eindruck, daß das Verleihen von Bankettausstattungen bemerkenswert war. Man müßte ihn dann, wenn auch nicht für unhistorisch, so doch für irreführend halten, wenn sich Polykrates dabei so verhielt wie jeder andere Adlige auch. ${ }^{29}$ Um die Historizität der Geschichte scheint es allerdings nicht allzu schlecht bestellt zu sein..$^{30}$ Es überrascht, daß die Geschichte explizit in die Zeit vor der Tyrannis datiert wird: Ein Erfinder hätte sie erwartungsgemäß gerade in die Zeit der Tyrannis fallen lassen. Für deren Historizität spricht auch der Umstand, daß die Erzählung keinem starren Schema folgt, vielmehr bahnt sich Polykrates einen eigenen

\footnotetext{
${ }^{27}$ Gow 1952b: 298-99, 300 u. A. 1.

${ }^{28}$ Bereits Ure (1922: 79) fällte über seine „coverlets“ ein ähnliches Urteil: „The word seems to denote a Samian specialty."

${ }^{29}$ Ein Gutachter zweifelte nicht an der Historizität der Geschichte, sondern vertrat die Ansicht, daß das Leihverfahren weit verbreitet und auch im republikanischen Rom anzutreffen sei; er bzw. sie verwies dabei auf Plin. NH 33.143: invenimus legatos Carthaginiensium dixisse nullos hominum inter sese benignius vivere quam Romanos. eodem enim argento apud omnes cenitavisse ipsos. Aus dem Zusammenhang geht jedoch hervor, daß dies im weiteren Verlauf der republikanischen Geschichte anders war.

${ }^{30}$ Im Gegensatz etwa zu den Tierimporten aus der Zeit der Tyrannis, deren Historizität er bestritt, bringt Bernhardt (2003: 29-30) keine Einwände gegen die „Draperien...und Becher“ vor.
} 
Weg nach oben. ${ }^{31}$ Die Wahrscheinlichkeit der Nachricht erhöht zudem der Umstand, daß Polykrates in einer Zeit lebte, da sich häusliche Bankette noch in der Entwicklung befanden. Freilich entstand der Brauch, im Liegen zu speisen, im späteren 7. Jahrhundert, ${ }^{32}$ dieser Brauch wurde aber "offenbar noch nicht überall und zu jeder Gelegenheit" gepflegt. ${ }^{33}$ So wurde das Bett möglicherweise erst im Laufe der Zeit mit einer Armlehne versehen und so zum richtigen Speisebett gemacht. ${ }^{34}$ Tatsächlich belegen zwei Zeitgenossen von Polykrates weiterhin den Brauch, im Sitze zu speisen und trinken. So empfiehlt Theognis von Megara, mit guten Männern zu trinken, essen, und sit-

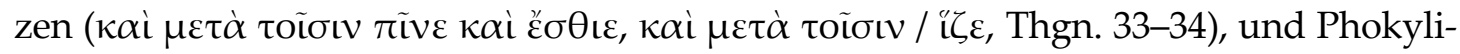
des aus Milet seinerseits, daß der Gast im Sitzen plaudert und seinen Wein trinkt ( $\chi \varrho \eta$

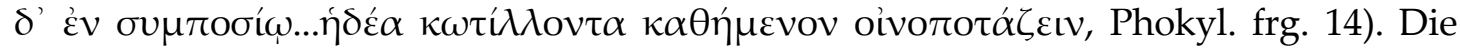
Sitte des Liegens beim Mahle herrschte also zu Lebzeiten des Polykrates (gest. um 522/1) nicht einmal in Ionien ausnahmslos. Ob die Vorlage des Athenaios weiteren Aufschluß gab, können wir nicht wissen. Die Erzählung von den ausgeliehenen $\sigma \tau \varrho \omega \mu v \alpha i ́$ und

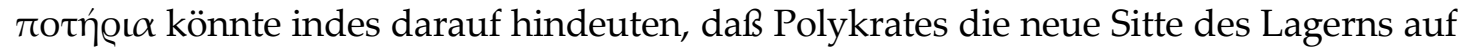
Samos einführte. Denkbar wäre auch, daß Polykrates zwar nicht die Sitte selbst, aber doch das eigens dafür entwickelte Möbelstück einführte. Wer die Worte von Theognis und Phokylides in Erinnerung behält, der wird dem Privatmann Polykrates derartige Leistungen nicht so schnell absprechen wollen.

\author{
Dr. F. X. Ryan \\ Los Angeles, California \\ E-mail:fxryan@gmx.de
}

\footnotetext{
${ }^{31}$ Es ist kein zu gewagter Schluß mit Ure-sieht man von seiner Auffassung der $\sigma \tau \varrho \omega \mu \nu \alpha i ́$ ab-anzunehmen: „It could scarcely be more definitely stated that Polycrates owed his throne to his wealth in coverlets and drinking vessels."

32 Vgl. Fehr (1971: 26), der folgerte, „daß man bereits im späten 7. Jahrhundert das Zechen auf Klinen kannte“; West (1997: 32): „This custom only established itself among them in the last quarter of the seventh century."

${ }^{33}$ Fehr 1971: 26. Bei den Makedonern mußte ein Mann solange zu Tisch sitzen, bis er einen Eber ohne Netz durchbohrt hatte (Athen. 1.18a, cf. 17f).

${ }^{34}$ Vgl. dazu Boardman (1990: 122): „possibly...using...their best bedroom furniture, until the point was reached at which the furniture was made specifically for the feast rather than for sleeping." Die so veränderte Kline hatte laut Boardman (ebd., 126) ",a raised head-end, which, I suspect, facilitated its use more as a couch for feasting, where support for the elbow and upper body was required, than for sleeping." Die Kline mit erhöhtem Kopfteil ist nach Kyrieleis (1969: 124) „,von der zweiten Hälfte des 6. Jahrhunderts bis ins späte 4. Jahrhundert zu verfolgen."
} 


\section{Literaturverzeichnis}

Bernhardt, R. (2003) Luxuskritik und Aufwandsbeschränkungen in der griechischen Welt. Stuttgart: Steiner.

Berve, H. (1967) Die Tyrannis bei den Griechen. München: Beck.

Boardman, J. (1990) ,Symposion Furniture.' - Murray, O. (ed.), Sympotica: A Symposium on the Symposion. Oxford: Clarendon Press, 122-31.

Burton, J. B. (1995) Theocritus's Urban Mimes. Berkeley: University of California Press.

Dentzer, J.-M. (1982) Le motif du banquet couché dans le Proche-Orient et le monde grec du VII ${ }^{e}$ au IV e siècle avant J.-C. Roma: École française de Rome.

Fehr, B. (1971) Orientalische und Griechische Gelage. Bonn: Bouvier Verlag Herbert Grundmann.

Fornara, C. W. (1983) Archaic Times to the End of the Peloponnesian War. Cambridge: Cambridge University Press.

Friedrich, C. (2000) Athenaios, Das Gelehrtenmahl. Buch XI-XIII. Stuttgart: Anton Hiersemann.

Galvagno, E. (1994) ,L'economia del tiranno: il caso di Policrate di Samo.' - RSA 24, 747.

Gerber, D. E. (1997) ,Callinus.' - Gerber, D. E. (ed.), A Companion to the Greek Lyric Poets. Leiden: Brill, 99-101.

Gow, A. S. F. (1952) Theocritus. 2nd ed. 1952a: Vol. 1. 1952b: Vol. 2. Cambridge: Cambridge University Press.

Gulick, C. B. (1933) Athenaeus, The Deipnosophists, Books XI-XII. Cambridge, Mass: Loeb Classical Library.

Harrison, J. A. (1893) The Story of Greece. New York: G. P. Putnam's Sons.

Kyrieleis, H. (1969) Throne und Klinen. Studien zur Formgeschichte altorientalischer und griechischer Sitz- und Liegemöbel vorhellenistischer Zeit. Berlin: de Gruyter.

Lenschau, Th. (1952) ,Polykrates 1.' - RE 21.2, 1726-34.

Libero, L. de (1996) Die archaische Tyrannis. Stuttgart: Steiner.

Miller, A. M. (1996) Greek Lyric: An Anthology in Translation. Indianapolis: Hackett.

Müller, K. O. (1882) Geschichte der griechischen Literatur bis auf das Zeitalter Alexanders. Bd. 1. Stuttgart: Albert Heitz.

Plaß, H. G. (1859) Die Tyrannis in ihren beiden Perioden bei den alten Griechen. 2., unveränderte Ausg. Leipzig: Adolf Gumprecht.

Ploß, H. (1884) Das Kind in Brauch und Sitte der Völker. Anthropologische Studien. 2. Bd., 2. Aufl. Leipzig: Th. Grieben's Verlag (L. Fernau).

Rawlinson, G.; Rawlinson, H.; Wilkinson, J. G. (1862) History of Herodotus. Vol. 2. London: John Murray.

Richter, G. M. A. (1966) The Furniture of the Greeks, Etruscans and Romans. London: Phaidon Press.

Sewell, E. M. (1862) Ancient History of Egypt, Assyria, and Babylonia. London: Longman, Green, Longman, and Roberts.

Shipley, G. (1987) A History of Samos, 800-188 BC. Oxford: Clarendon Press.

Stahl, M. (1987) Aristokraten und Tyrannen im archaischen Athen. Untersuchungen zur Überlieferung, zur Sozialstruktur und zur Entstehung des Staates. Wiesbaden: Franz Steiner.

Staiger, E. (1970) Theokrit. Die echten Gedichte. Zürich: Artemis. 
Thompson, D. B. (1988) Swans and Amber: Some Early Greek Lyrics Freely Translated and Adapted. [Zuerst 1948.] Princeton: ASCSA.

Ure, P. N. (1922) The Origin of Tyranny. Cambridge: University Press.

Verity, A. (2002) Theocritus, Idylls. Oxford: Oxford University Press.

Vössing, K. (2004) Mensa regia. Das Bankett beim hellenistischen König und beim römischen Kaiser. München: Saur.

West, M. L. (1997) The East Face of Helicon: West Asiatic Elements in Greek Poetry and Myth. Oxford: Clarendon Press.

Westgate, R. (1997-1998) ,Greek Mosaics in their Architectural and Social Context.' BICS 42, 93-115.

Yonge, C. D. (1854) The Deipnosophists or Banquet of the Learned of Athenaeus. Vol. 3. London: Henry G. Bohn. 